\title{
Finite-Time Stability Analysis and Control for a Class of Stochastic Singular Biological Economic Systems Based on T-S Fuzzy Model
}

\author{
Shuangyun Xing, ${ }^{1,2}$ Qingling Zhang, ${ }^{1}$ and Yi Zhang ${ }^{3}$ \\ ${ }^{1}$ Institute of Systems Science, Northeastern University, Shenyang, Liaoning 110189, China \\ ${ }^{2}$ College of Science, Shenyang Jianzhu University, Shenyang, Liaoning 110168, China \\ ${ }^{3}$ School of Science, Shenyang University of Technology, Shenyang, Liaoning 110870, China \\ Correspondence should be addressed to Qingling Zhang; qlzhang@mail.neu.edu.cn
}

Received 28 February 2013; Accepted 23 May 2013

Academic Editor: Qun Lin

Copyright ( 2013 Shuangyun Xing et al. This is an open access article distributed under the Creative Commons Attribution License, which permits unrestricted use, distribution, and reproduction in any medium, provided the original work is properly cited.

\begin{abstract}
This paper studies the problem of finite-time stability and control for a class of stochastic singular biological economic systems. It shows that such systems exhibit the distinct dynamic behavior when the economic profit is a variable rather than a constant. Firstly, the stochastic singular biological economic systems are established as fuzzy models based on T-S fuzzy control approach. These models are described by stochastic singular T-S fuzzy systems. Then, novel sufficient conditions of finite-time stability are obtained for the stochastic singular biological economic systems, and the state feedback controller is designed so that the population (state of the systems) can be driven to the bounded range by the management of the open resource. Finally, by using Matlab software, numerical examples are given to illustrate the effectiveness of the obtained results.
\end{abstract}

\section{Introduction}

At present, mankind is facing the problems of shortage of resources and worsening environment. So there has been rapidly growing interest in the analysis and modeling of biological systems. From the view of human needs, the exploitation of biological resources and harvest of population are commonly practiced in the fields of fishery, wildlife, and forestry management, which has already been tackled in the classical literature (see [1-5]). In these works, the main objective was to maximize a utility function representing the sustainable economic rent (i.e., net economic income). The results may not be realistic due to assumptions under which the models are studied. Indeed, the validity of the assumption stipulating that price is constant seems to be limited to a small-scale fishery, wildlife, or forestry. Also, the assumption of free access to valuable resources must be discarded because it inevitably leads to an overexploitation. For instance, the Moroccan octopus crisis arising between 1996 and 1997 generated a biological (overexploitation of the resource) and economic instability (raising the resource price).

To avoid these situations, Jerry and Raïssi [6] have studied the biological and economic stabilizability by adding a dynamic prevailing on the market in order to take into account resource price variations based on a standard openaccess fisheries model (see [1,7-10]); the model was given by

$$
\begin{gathered}
d x(t)=\left[r x(t)\left(1-\frac{x(t)}{k}\right)-E x(t)\right] d t, \\
d p(t)=[s(a-p(t)-E x(t))] d t,
\end{gathered}
$$

where $x(t)$ is the population densities at time $t, p(t)$ is the unit price of the population at time $t$. The constants $r>0, k>0$ are the intrinsic growth rate and the carrying capacity of the population, respectively. $E>0$ is constant representing harvesting effort. $a$ is a positive constant parameter, representing the market capacity. $s$ is the price speed adjustment, and it reflects market competition (i.e., perfect elasticity of price). 
In daily life, economic profit is a very important factor for governments, merchants, and even every citizen, so it is necessary to investigate biological economic systems, which are always described by differential-algebraic equations. At present, most of differential-algebraic equations can be found in the general power systems [11, 12], economic administration [13], robot system [14], mechanical engineering [15], and so on. However, to the best of our knowledge, the reports of such systems are few in biology. Considering the economic theory of fishery resource [16] proposed by Gordon in 1954, this paper studies a class of singular biological economic model as follows:

$$
\begin{gathered}
d x(t)=\left[r x(t)\left(1-\frac{x(t)}{k}\right)-E x(t)\right] d t, \\
d p(t)=[s(a-p(t)-E x(t))] d t, \\
0=E p(t) x(t)-c E x(t)-m(t),
\end{gathered}
$$

where $c>0$ is harvesting cost per unit harvesting effort for the population, $m(t) \geq 0$ is the economic profit per unit harvesting effort at time $t$. From the previous modeling view, the economic profit is variable. In order to facilitate the analysis, many authors assume that the economic profit is constant (see [17-20]). In fact, the economic profit is not always constant and may vary with numerous factors, such as seasonality, revenue, market demand, and harvesting cost and so on. Hence, it is more reasonable that the economic profit is a variable from real world point of view, and model (2) is more suitable for the analysis of the dynamic behavior of biological economic systems. The economic profit is affected by the unit price of the population, and the population densities of our discussed model and other factors are omitted. However, owing to difficulty arising in analysis, few results are concerned with such systems.

It is well known that the white noise always exists and we cannot omit the influence of the white noise to many dynamical systems. In reality, due to continuous fluctuations in the environment (e.g., variation in intensity of sunlight, temperature, water level, etc.), parameters involved in models are not absolute constants, but they always fluctuate around some average value. As a result, the population density never attains a fixed value with the advancement of time but rather exhibits continuous oscillation around some average values. Based upon these factors, stochastic population models have received more and more attention [21-23]. Many authors studied the effect of the stochastic perturbation to the biological economic system with different functional responses, such as [24-27]. However, only a small amount of work has been studied for the stochastic perturbation biological economic systems with differential-algebraic equations. In this paper, we introduce stochastic perturbation to system (2) and obtain the following stochastic singular system:

$$
\begin{gathered}
d x(t)=\left[r x(t)\left(1-\frac{x(t)}{k}\right)-E x(t)\right] d t+\sigma x(t) d W(t), \\
d p(t)=s(a-p(t)-E x(t)), \\
0=E p(t) x(t)-c E x(t)-m(t),
\end{gathered}
$$

where $W(t)$ is one-dimensional Brownian motion with $W(0)=0$ and $\sigma^{2}$ is intensities of the white noise.

The proposed system (3) has a more widely practical background in this paper. For example, we show that there is a stationary distribution of system (3) if the white noise is small. While if the white noise is large, we prove that the prey population will either extinct or its distribution converges to a probability measure. So, we may study the economic profit through the changes of population density and the variation of market price in the stochastic environment. For this reason, we say the stochastic model is more realistic than the deterministic model.

The aim of this paper is to discuss the finite-time stability and control problem of the system (2) and (3). It is now worth pointing out that the stability, stabilization, and control performance mentioned previously concern the desired behavior of the controlled dynamic systems over an infinite-time interval, which always deals with the asymptotic property of system trajectories [28-30]. But in some practical processes, the asymptotically stable system over an infinite-time interval does not mean that it has good transient characteristics, for instance, biochemistry reaction system, robot control system, communication network system, and so forth. Therefore, it is necessary to study the transient behavior over a finite-time interval.

On the other hand, the literature on finite-time stability (FTS) (or short-time stability) of systems has attracted particular interests of researchers. Some earlier results on finitetime stability date back to the 1960s [31]. Recently, the concept of finite-time stability has been revisited in the light of linear matrix inequality theory. Several sufficient conditions for finite-time stability and stabilization of continuous time systems or discrete time systems have been presented [32, 33]. References $[34,35]$ have extended the definition of finite-time stability to linear systems with impulsive effects or singular systems with impulsive effects, respectively, and derived some sufficient conditions for finite-time stability and stabilization problems. Very recently, [36] generalized the results of [32] to linear stochastic systems. However, to date and to the best of our knowledge, no work so far has been done on the problem of finite-time stability for a class of stochastic singular biological economic systems. The problems are important in many practice applications, which motivate the main purpose of our study.

T-S fuzzy model, proposed by Takagi and Sugeno [37] in 1985, has been shown to be a powerful tool for modeling complex nonlinear systems. It is wellknown that, by means of the T-S fuzzy model, a nonlinear system can be represented by a weighted sum of some simple linear subsystems and then can be stabilized by a model-based fuzzy control. Therefore, many issues related to the stability analysis of the complex nonlinear systems can be discussed using the T-S fuzzy model.

In this paper, we investigate the problem of finitetime stability and control for stochastic singular biological economic systems. Our results are totally different from those previous results. Firstly, the system (2) and (3) are established for fuzzy models based on T-S fuzzy control approach. Then, sufficient conditions of finite-time stability are obtained for 
the system (2) and (3). One of the contributions of this paper is that the economic profit, which is a variable rather than a constant of the biological economic systems, is discussed. We study the economic profit fluctuations through the unit price range and the population changes. The other contribution is to study the finite-time stability for the biological economic system with stochastic perturbation, and sufficient criterions are presented for the solvability of the problem, which can be reduced to a feasibility problem involving restricted linear matrix, inequalities with a fixed parameter. Based on this, the state feedback controller is designed so that the biological population can be controlled in the bounded range by the management of the open resource. The corresponding sufficient conditions are given as well. Finally, some numerical simulations on feasible region are given to illustrate the effectiveness of our approach.

The rest of the paper is organized as follows. In Section 2, fuzzy modeling and preliminaries are stated in this paper. In Section 3, the finite-time stability of stochastic biological economic systems is studied, which provides some sufficient conditions for systems to be FTS. Meanwhile, theorems of sufficient conditions for FTS are established. In Section 4, finite-time controllers are designed for the stochastic biological economic systems. Numerical examples are provided in Section 5. Conclusions are given in Section 6.

Notations. In this paper, the superscript “ $T$ ” stands for matrix transposition. The notation $X>0$ means $X$ is a positive definite matrix. $\varepsilon(x)$ denotes the expectation of stochastic variable $x \cdot \operatorname{deg}(\cdot)$ denotes the degree of the determinant. In addition, the symbol “ $*$ " denotes the transposed elements in the symmetric positions of a matrix and $\operatorname{diag}(\cdot)$ stands for a block-diagonal matrix. $\lambda_{\text {min }}(P)$ and $\lambda_{\text {max }}(P)$ denote the smallest and the largest eigenvalue of matrix $P$, respectively.

\section{Fuzzy Modeling and Preliminaries}

Considering the model (2), for simplicity, we use the following coordinate transformation

$$
\xi(t)=p(t)-a
$$

Then, the model (2) can be rewritten as follows:

$$
\begin{gathered}
d x(t)=\left[r x(t)\left(1-\frac{x(t)}{k}\right)-E x(t)\right] d t, \\
d \xi(t)=[-s E x(t)-s \xi(t)] d t \\
0=(a-c) E x(t)+E x(t) \xi(t)-m(t) .
\end{gathered}
$$

Let $X(t)=[x(t) \xi(t) m(t)]^{\mathrm{T}}$; the previous model can be expressed by fuzzy model, which consists of the following two rules:

$$
\begin{aligned}
& R^{1}: \text { If } x(t) \text { is } F_{1}, \text { then } \Xi \dot{X}(t)=A_{1} X(t) \\
& R^{2}: \text { If } x(t) \text { is } F_{2}, \text { then } \Xi \dot{X}(t)=A_{2} X(t),
\end{aligned}
$$

where $\Xi=\left[\begin{array}{lll}1 & 0 & 0 \\ 0 & 1 & 0 \\ 0 & 0 & 0\end{array}\right], A_{1}=\left[\begin{array}{ccc}2 r-E & 0 & 0 \\ -s E & -s & 0 \\ a E-c E & -k E & -1\end{array}\right], A_{2}=\left[\begin{array}{ccc}-E & 0 & 0 \\ -s E & -s & 0 \\ a E-c E & k E & -1\end{array}\right]$.
$F_{i} \quad(i=1,2)$ is the fuzzy set, $h_{i}(x(t)) \geq 0$ is a membership function of $F_{i}$, and $h_{1}(x(t))=(1 / 2)(1-(x(t) / k)), h_{2}(x(t))=$ $(1 / 2)(1+(x(t) / k))$, and $\sum_{i=1}^{2} h_{i}(x(t))=1$.

By using a standard fuzzy singleton inference method, a singleton fuzzifier to produce a fuzzy inference, and a weighted center-average defuzzifier, we can obtain the global fuzzy system as follows:

$$
\Xi \dot{X}(t)=A_{h} X(t)
$$

where $A_{h}=\sum_{i=1}^{2} h_{i}(x(t)) A_{i}$.

As for the model (3), using the similar method, we can obtain

$$
\Xi d X(t)=A_{h} X(t) d t+C X(t) d W(t)
$$

where $C=\left[\begin{array}{lll}\sigma & 0 & 0 \\ 0 & 0 & 0 \\ 0 & 0 & 0\end{array}\right]$

Definition 1. (i) The pair $\left(\Xi, A_{h}\right)$ is said to be regular if $\operatorname{det}\left(\lambda \Xi-A_{h}\right)$ is not identically zero.

(ii) The pair $\left(\Xi, A_{h}\right)$ is said to be impulse free if $\operatorname{deg}\left(\operatorname{det}\left(\lambda \Xi-A_{h}\right)\right)=\operatorname{rank}(\Xi)$.

Remark 2. In this paper, through the calculation, it is known that the pair $\left(\Xi, A_{h}\right)$ is regular.

Definition 3. Given two positive real numbers $c_{1}, c_{2}$ with $c_{1} \leq$ $c_{2}$, a positive definite matrix $R$, system (7) is said to be finitetime stable with respect to $\left(c_{1}, c_{2}, T, R\right)$ if

$$
\begin{array}{r}
X^{\mathrm{T}}(0) \Xi^{\mathrm{T}} R \Xi X(0) \leq c_{1} \Longrightarrow X^{\mathrm{T}}(t) \Xi^{\mathrm{T}} R \Xi X(t)<c_{2}, \\
\forall t \in[0, T] .
\end{array}
$$

Definition 4. Given two positive real numbers $c_{1}, c_{2}$ with $c_{1} \leq c_{2}$, a positive definite matrix $R$, system (8) is said to be stochastic finite-time stable with respect to $\left(c_{1}, c_{2}, T, R\right)$ if

$$
\begin{array}{r}
\varepsilon\left\{X^{\mathrm{T}}(0) \Xi^{\mathrm{T}} R \Xi X(0)\right\} \leq c_{1} \Longrightarrow \varepsilon\left\{X^{\mathrm{T}}(t) \Xi^{\mathrm{T}} R \Xi X(t)\right\}<c_{2}, \\
\forall t \in[0, T] .
\end{array}
$$

Remark 5. Asymptotic stability and FTS are independent concepts of each other as it was described in [31]. In certain cases, asymptotic stability can be also considered as an additional requirement while restricting our attention over a finite-time interval. The definition of FTS can be interpreted in terms of ellipsoidal domains. The set defined by $\left\{X^{\mathrm{T}}(0) \Xi^{\mathrm{T}} R \Xi X(0) \leq c_{1}\right\}$ contains all the admissible initial states. Instead, the inequality $\left\{X^{\mathrm{T}}(t) \Xi^{\mathrm{T}} R \Xi X(t)<c_{2}\right\}$ defines a time-varying ellipsoid which bounds the state trajectory over the finite-time interval $t \in[0, T]$. In addition, we can fix the upper bound beforehand in finite-time control, and in general, the initial and ultimates bound are not the same. Thus, this concept will be more meaningful in practice, such as aircraft control, networked control, and robot operating. 
Definition 6 (see [38]). Let $V(x(t), t)$ be the stochastic Lyapunov function of the stochastic system, where $x(t)$ satisfies the following stochastic differential equation:

$$
d x(t)=f(t) d t+g(t) d W(t) .
$$

Define a weak infinitesimal operator $\mathscr{L}$ of random process $\{x(t), t>0\}$ :

$$
\begin{aligned}
\mathscr{L} V(x(t), t)= & V_{t}(x(t), t)+V_{x}(x(t), t) f(t) \\
& +\frac{1}{2} \operatorname{tr}\left[g^{\mathrm{T}}(t) V_{x x}(x(t), t) g(t)\right] .
\end{aligned}
$$

Lemma 7 (see [39], Gronwalls inequality). Let $v(t)$ be a nonnegative function such that

$$
v(t) \leq a+b \int_{0}^{t} v(s) d s, \quad 0 \leq t \leq T,
$$

for some constants $a, b \geq 0$, then we have

$$
v(t) \leq a \exp (b t), \quad 0 \leq t \leq T .
$$

Lemma 8 (Schur's complement). For real matrices $N, M^{T}=$ $M$, and $R=R^{T}>0$, the following three conditions are equivalent:

(1) $M+N R^{-1} N^{T}<0$,

(2) $\left(\begin{array}{cc}M & N \\ N^{T} & -R\end{array}\right)<0$,

(3) $\left(\begin{array}{cc}M & -N \\ -N^{T} & -R\end{array}\right)<0$.

Lemma 9 (see [40]). The following items are true.

(i) Assume that $\operatorname{rank}(\Xi)=r$, there exist two orthogonal matrices $U$ and $V$ such that $\Xi$ has the decomposition as

$$
\Xi=U\left[\begin{array}{cc}
\Sigma_{r} & 0 \\
* & 0
\end{array}\right] V^{T}=U\left[\begin{array}{cc}
I_{r} & 0 \\
* & 0
\end{array}\right] v^{T}
$$

where $\Sigma_{r}=\operatorname{diag}\left\{\delta_{1}, \delta_{2}, \ldots, \delta_{r}\right\}$ with $\delta_{k}>0$ for all $k=$ $1,2, \ldots, r$. Partition $U=\left[\begin{array}{ll}U_{1} & U_{2}\end{array}\right], V=\left[\begin{array}{ll}V_{1} & V_{2}\end{array}\right]$ and $v=\left[\begin{array}{ll}V_{1} \Sigma_{r} & V_{2}\end{array}\right]$ with $\Xi V_{2}=0$ and $U_{2}^{\mathrm{T}} \Xi=0$.

(ii) If $P$ satisfies

$$
\Xi P^{\mathrm{T}}=P \Xi^{\mathrm{T}} \geq 0
$$

Then $\widetilde{P}=U^{\mathrm{T}} P v$ with $U$ and $v$ satisfying (8) if and only if

$$
\widetilde{P}=\left[\begin{array}{cc}
P_{11} & P_{12} \\
0 & P_{22}
\end{array}\right]
$$

with $P_{11} \geq 0 \in \mathbb{R}^{r \times r}$. In addition, when $P$ is nonsingular, we have $P_{11}>0$ and $\operatorname{det}\left(P_{22}\right) \neq 0$. Furthermore, $P$ satisfying (16) can be parameterized as

$$
P=\Xi v^{-\mathrm{T}} Y v^{-1}+U Z V_{2}^{\mathrm{T}},
$$

where $Y=\operatorname{diag}\left\{P_{11}, \Psi\right\}, Z=\left[\begin{array}{ll}P_{12}^{\mathrm{T}} & P_{22}^{\mathrm{T}}\end{array}\right]^{\mathrm{T}}$, and $\Psi \in$ $\mathbb{R}^{(n-r) \times(n-r)}$ is an arbitrary parameter matrix. (iii) If $P$ is a nonsingular matrix, $R$ and $\Psi$ are two positive definite matrices, $P$ and $\Xi$ satisfy (16), $Y$ is a diagonal matrix from (18), and the following equality holds:

$$
P^{-1} \Xi=\Xi^{\mathrm{T}} R^{1 / 2} Q R^{1 / 2} \Xi .
$$

Then the positive definite matrix $Q=R^{-1 / 2} U Y^{-1} U^{T}$ $R^{-1 / 2}$ is a solution of (19).

\section{FTS of the Stochastic Singular Biological Economic System}

This section provides finite-time stability analysis results for the stochastic singular biological economic system, some novel sufficient conditions for finite-time stability of the stochastic singular system are given. Through the previous analysis, we know that the system (2) and (3) are equivalent to the system (7) and (8), respectively. Because the system (7) and (8) are regular, the following theorems are given.

\subsection{FTS of the System (7)}

Theorem 10. For a given time constant $T>0$ and a scalar $\alpha>$ 0 , the system (7) is finite-time stable with respect to $\left(c_{1}, c_{2}, T, R\right)$ if there exist a nonsingular matrix $P$ and a symmetric positive definite matrix $Q$ such that the following conditions hold:

$$
\begin{gathered}
P \Xi^{\mathrm{T}}=\Xi P^{\mathrm{T}} \geq 0, \\
A_{1} P^{\mathrm{T}}+P A_{1}^{\mathrm{T}}-\alpha \Xi P^{\mathrm{T}}<0, \\
A_{2} P^{\mathrm{T}}+P A_{2}^{\mathrm{T}}-\alpha \Xi P^{\mathrm{T}}<0, \\
P^{-1} \Xi=\Xi^{\mathrm{T}} R^{1 / 2} Q R^{1 / 2} \Xi, \\
\lambda_{\max }(Q) c_{1} e^{\alpha T}-c_{2} \lambda_{\min }(Q)<0 .
\end{gathered}
$$

Proof. Consider the following Lyapunov function:

$$
V(X(t), t)=X^{\mathrm{T}}(t) P^{-1} \Xi X(t) .
$$

We first prove that the following condition (25) is equivalent to (21a) and (21b):

$$
\dot{V}(X(t), t)<\alpha V(X(t), t) .
$$

When $t \in[0, T]$, we calculate the time derivative of $V(X(t), t)$ along the solution of system (7) and obtain

$$
\dot{V}(X(t), t)=X^{\mathrm{T}}(t)\left(A_{h}{ }^{\mathrm{T}} P^{-\mathrm{T}}+P^{-1} A_{h}\right) X(t)
$$

which leads to

$$
\begin{aligned}
\dot{V} & (X(t), t)-\alpha V(X(t), t) \\
& =X^{\mathrm{T}}(t)\left(A_{h}{ }^{\mathrm{T}} P^{-\mathrm{T}}+P^{-1} A_{h}-\alpha P^{-1} \Xi\right) X(t) .
\end{aligned}
$$


So, (27) is equivalent to the following inequality

$$
A_{h}{ }^{\mathrm{T}} P^{-\mathrm{T}}+P^{-1} A_{h}-\alpha P^{-1} \Xi<0 .
$$

Pre- and postmultiplying (28) by $P$ and $P^{\mathrm{T}}$, we get

$$
A_{h} P^{\mathrm{T}}+P A_{h}^{\mathrm{T}}-\alpha \Xi P^{\mathrm{T}}<0 .
$$

When $A_{h}=\sum_{i=1}^{2} h_{i}(x(t)) A_{i}$, through the matrix decomposition, we can see that (29) is equivalent to (21a) and (21b).

Thus, dividing by $V(X(t), t)$ on both sides of (25) and integrating it from 0 to $t$ with $t \in[0, T]$, one gets

$$
V(X(t), t)<V(X(0), 0) e^{\alpha t} .
$$

By condition (22), one can deduce that

$$
\begin{aligned}
V(X(t), t) & =X^{\mathrm{T}}(t) P^{-1} \Xi X(t) \\
& =X^{\mathrm{T}}(t) \Xi^{\mathrm{T}} R^{1 / 2} Q R^{1 / 2} \Xi X(t) \\
& \geq \lambda_{\min }(Q) X^{\mathrm{T}}(t) \Xi^{\mathrm{T}} R \Xi X(t), \\
V(x(0), 0) e^{\alpha t} & =X^{\mathrm{T}}(0) \Xi^{\mathrm{T}} R^{1 / 2} Q R^{1 / 2} \Xi X(0) e^{\alpha t} \\
& \leq \lambda_{\max }(Q) X^{\mathrm{T}}(0) \Xi^{\mathrm{T}} R \Xi X(0) e^{\alpha t} \\
& \leq \lambda_{\max }(Q) c_{1} e^{\alpha T} .
\end{aligned}
$$

From (31), it is easy to obtain

$$
X^{\mathrm{T}}(t) \Xi^{\mathrm{T}} R \Xi X(t) \leq \frac{\lambda_{\max }(Q)}{\lambda_{\min }(Q)} c_{1} e^{\alpha T} .
$$

Considering condition (23) and inequality (32), it is easy to get $X^{\mathrm{T}}(t) \Xi^{\mathrm{T}} R \Xi X(t)<c_{2}$ for all $t \in[0, T]$. This completes the proof of the theorem.

\subsection{FTS of the System (8)}

Theorem 11. For a given time constant $T>0$ and a scalar $\alpha>0$, the system (8) is stochastic finite-time stable with respect to $\left(c_{1}, c_{2}, T, R\right)$ if there exist a nonsingular matrix $P$ and $a$ symmetric positive definite matrix $Q$ such that (20), (22), and (23) hold and the following matrix inequalities are satisfied:

$$
\begin{gathered}
{\left[\begin{array}{cc}
A_{1} P^{\mathrm{T}}+P A_{1}^{\mathrm{T}}-\alpha \Xi P^{\mathrm{T}} & \left(\Xi C P^{\mathrm{T}}\right)^{\mathrm{T}} \\
\Xi C P^{\mathrm{T}} & -\widetilde{\mathrm{Q}}
\end{array}\right]<0,} \\
{\left[\begin{array}{cc}
A_{2} P^{\mathrm{T}}+P A_{2}^{\mathrm{T}}-\alpha \Xi P^{\mathrm{T}} & \left(\Xi C P^{\mathrm{T}}\right)^{\mathrm{T}} \\
\Xi C P^{\mathrm{T}} & -\widetilde{\mathrm{Q}}
\end{array}\right]<0,}
\end{gathered}
$$

where $\widetilde{Q}=R^{-1 / 2} Q^{-1} R^{-1 / 2}$.

Proof. Let us consider the quadratic Lyapunov function

$$
V(X(t), t)=X^{\mathrm{T}}(t) P^{-1} \Xi X(t) .
$$

Let $\mathscr{L}$ be the infinitesimal generator (also called the averaged derivative). We first show the following condition:

$$
\mathscr{L} V(X(t), t)<\alpha V(X(t), t) .
$$

Now we will prove that condition (35) is equivalent to (33a) and (33b). Applying Ito's formula, it follows that

$$
\begin{aligned}
& \mathscr{L V}(X(t), t) \\
& \quad=X^{\mathrm{T}}(t)\left(A_{h}^{\mathrm{T}} P^{-\mathrm{T}}+P^{-1} A_{h}+C^{\mathrm{T}} P^{-1} \Xi C\right) X(t)
\end{aligned}
$$

which leads to

$$
\begin{aligned}
\mathscr{L} V(X(t), t) & -\alpha V(X(t), t) \\
=X^{\mathrm{T}}(t)( & A_{h}^{\mathrm{T}} P^{-\mathrm{T}} \\
& \left.+P^{-1} A_{h}+C^{\mathrm{T}} P^{-1} \Xi C-\alpha P^{-1} \Xi\right) X(t) .
\end{aligned}
$$

Combining condition (22) with $\widetilde{Q}=R^{-1 / 2} Q^{-1} R^{-1 / 2}$, we can obtain

$$
\begin{aligned}
\mathscr{L} V(X(t), t)-\alpha V(X(t), t) & \\
=X^{\mathrm{T}}(t) & \left(A_{h}^{\mathrm{T}} P^{-\mathrm{T}}+P^{-1} A_{h}\right. \\
& \left.+C^{\mathrm{T}} \Xi^{\mathrm{T}} \widetilde{Q}^{-1} \Xi C-\alpha P^{-1} \Xi\right) X(t) .
\end{aligned}
$$

So, (37) is equivalent to

$$
A_{h}^{\mathrm{T}} P^{-\mathrm{T}}+P^{-1} A_{h}+C^{\mathrm{T}} \Xi^{\mathrm{T}} \widetilde{Q}^{-1} \Xi C-\alpha P^{-1} \Xi<0 .
$$

Pre- and postmultiplying (39) by $P$ and $P^{\mathrm{T}}$, we get

$$
P A_{h}^{\mathrm{T}}+A_{h} P^{\mathrm{T}}+P C^{\mathrm{T}} \Xi^{\mathrm{T}} \widetilde{Q}^{-1} \Xi C P^{\mathrm{T}}-\alpha \Xi P^{\mathrm{T}}<0 .
$$

When $A_{h}=\sum_{i=1}^{2} h_{i}(x(t)) A_{i}$, through the matrix decomposition and by Lemma 8 , we can see that (40) is equivalent to (33a) and (33b).

Hence, integrating both sides of (35) from 0 to $t$ with $t \epsilon$ $[0, T]$ and then taking the expectation, it yields

$$
\varepsilon\{V(X(t), t)\}<V(X(0), 0)+\alpha \int_{0}^{t} \varepsilon\{V(X(s), s)\} d s .
$$

By Lemma 7, we can obtain

$$
\varepsilon\{V(X(t), t)\}<V(X(0), 0) e^{\alpha t} .
$$

From (34), it follows that

$$
\begin{aligned}
\varepsilon\{V(X(t), t)\} & =\varepsilon\left\{X^{\mathrm{T}}(t) \Xi^{\mathrm{T}} R^{1 / 2} Q R^{1 / 2} \Xi X(t)\right\} \\
& \geq \lambda_{\min }(Q) \varepsilon\left\{X^{\mathrm{T}}(t) \Xi^{\mathrm{T}} R \Xi X(t)\right\}, \\
V(X(0), 0) e^{\alpha t} & =X^{\mathrm{T}}(0) \Xi^{\mathrm{T}} R^{1 / 2} Q R^{1 / 2} \Xi X(0) e^{\alpha t} \\
& \leq \lambda_{\max }(Q) X^{\mathrm{T}}(0) \Xi^{\mathrm{T}} R \Xi X(0) e^{\alpha t} \\
& \leq \lambda_{\max }(Q) c_{1} e^{\alpha T} .
\end{aligned}
$$


From (43), it is easy to obtain

$$
\varepsilon\left\{X^{\mathrm{T}}(t) \Xi^{\mathrm{T}} R \Xi X(t)\right\}<\frac{\lambda_{\max }(Q)}{\lambda_{\min }(Q)} c_{1} e^{\alpha T} .
$$

Considering condition (23) and inequality (44), it is easy to get $\varepsilon\left\{X^{\mathrm{T}}(t) \Xi^{\mathrm{T}} R \Xi X(t)\right\}<c_{2}$ for all $t \in[0, T]$. This completes the proof of the theorem.

\section{Finite-Time Control of the Stochastic Singular Biological Economic System}

Unstable fluctuations have always been regarded as unfavorable ones from the ecological managers' point of view. Meanwhile, price variation depends on the gap between the demand function and supply function. In order to plan harvesting strategies and maintain the sustainable development of system, it is necessary to take action to stabilize biological population and market directed economy. This paper proposes the following state feedback control method for the system (2) and (3):

$$
\begin{aligned}
& d x(t)= {\left[r x(t)\left(1-\frac{x(t)}{k}\right)-E x(t)+u(t)\right] d t, } \\
& d p(t)=s(a-p(t)-E x(t)) d t, \\
& 0= E p(t) x(t)-c E x(t)-m(t), \\
& d x(t)= {\left[r x(t)\left(1-\frac{x(t)}{k}\right)-E x(t)+u(t)\right] d t } \\
&+\sigma x(t) d W(t), \\
& d p(t)=s(a-p(t)-E x(t)) d t, \\
& 0=\operatorname{Ep}(t) x(t)-c E x(t)-m(t),
\end{aligned}
$$

where $u(t)$ is control variable, representing the management of the open resource.

Then, we use the similar T-S fuzzy method shown in Section 2 and consider the following state feedback fuzzy controller:

$$
u(t)=\sum_{i=1}^{2} h_{i}(x(t)) L_{i} X(t)
$$

where $L_{i}$ is the state feedback gain to be designed.

The model (45) and (46) can be expressed by the following fuzzy models:

$$
\begin{aligned}
\Xi \dot{X}(t) & =\sum_{i=1}^{2} h_{i}(x(t)) \sum_{j=1}^{2} h_{j}(x(t))\left(A_{i}+B L_{j}\right) X(t), \\
\Xi d X(t) & \\
= & \sum_{i=1}^{2} h_{i}(x(t)) \sum_{j=1}^{2} h_{j}(x(t)) \\
& \times\left[\left(A_{i}+B L_{j}\right) X(t) d t+C X(t) d W(t)\right],
\end{aligned}
$$

where $B=\left[\begin{array}{lll}1 & 0 & 0\end{array}\right]^{\mathrm{T}}$.
In the sequence, we provide finite-time stabilization results for above the mentioned closed-loop system (48) and (49). At the same time, some novel sufficient conditions for finite-time stabilization of the closed-loop singular biological economic system are given.

\subsection{Finite-Time Control of the System (45)}

Theorem 12. For a given time constant $T>0$ and a scalar $\alpha>0$, there exists a state feedback controller (47) such that the closed-loop system (48) is finite-time stable with respect to $\left(c_{1}, c_{2}, T, R\right)$ if there exist a nonsingular matrix $P$, a symmetric positive definite matrix $Q$, and matrix $Y_{j}, j=1,2$, such that (22), (23) hold, and the following matrix inequalities are satisfied:

$$
\begin{gathered}
P \Xi^{\mathrm{T}}=\Xi P^{\mathrm{T}} \geq 0, \\
\Phi_{i i}<0, \quad i=j, i, j=1,2, \\
\Phi_{i j}+\Phi_{j i}<0, \quad i<j, i, j=1,2,
\end{gathered}
$$

where $\Phi_{i j}=P A_{i}^{\mathrm{T}}+Y_{j}^{\mathrm{T}} B^{\mathrm{T}}+A_{i} P^{\mathrm{T}}+B Y_{j}-\alpha \Xi P^{\mathrm{T}}$. Then, the desired state feedback gain can be chosen as $L_{j}=Y_{j} P^{-\mathrm{T}}$.

Proof. Considering Theorem 10 and the closed-loop system (48), we can obtain the following matrix inequality

$$
\sum_{i=1}^{2} \sum_{j=1}^{2} h_{i}(x(t)) h_{j}(x(t)) \Pi_{i j}<0
$$

where $\Pi_{i j}=\left(A_{i}+B L_{j}\right) P^{\mathrm{T}}+P\left(A_{i}+B L_{j}\right)^{\mathrm{T}}-\alpha \Xi P^{\mathrm{T}}$.

Let $Y_{j}=L_{j} P^{\mathrm{T}}$. Then,

$$
\begin{aligned}
& \sum_{i=1}^{2} \sum_{j=1}^{2} h_{i}(x(t)) h_{j}(x(t)) \\
& \quad \times\left(P A_{i}^{\mathrm{T}}+Y_{j}^{\mathrm{T}} B^{T}+A_{i} P^{\mathrm{T}}+B Y_{j}-\alpha \Xi P^{\mathrm{T}}\right)<0 .
\end{aligned}
$$

Obviously, (53) is equivalent to

$$
\sum_{i=1}^{2} h_{i}^{2}(x(t)) \Phi_{i i}+\sum_{i=1}^{2} \sum_{j=1}^{2} h_{i}(x(t)) h_{j}(x(t))\left(\Phi_{i j}+\Phi_{j i}\right)<0 .
$$

The above inequality (54) is equivalent to (51a) and (51b).

Then, by a similar argument in the proof of Theorem 10 and using (22)-(23), we can easily get the above results. This completes the proof of the theorem.

\subsection{Finite-Time Control of the System (46)}

Theorem 13. For a given time constant $T>0$ and a scalar $\alpha>0$, there exists a state feedback controller (47) such that the closed-loop system (49) is stochastic finite-time stable with respect to $\left(c_{1}, c_{2}, T, R\right)$ if there exist a nonsingular matrix $P, a$ symmetric positive definite matrix $Q$, and matrix $Y_{j}, j=1,2$, 
such that (50), (22), and (23) hold, and the following matrix inequalities are satisfied:

$$
\begin{gathered}
{\left[\begin{array}{cc}
\Psi_{i i} & \left(\Xi C P^{\mathrm{T}}\right)^{\mathrm{T}} \\
\Xi C P^{\mathrm{T}} & -\widetilde{\mathrm{Q}}
\end{array}\right]<0 \quad i=j, i, j=1,2,} \\
{\left[\begin{array}{cc}
\Psi_{i j}+\Psi_{j i} & \left(\Xi C P^{\mathrm{T}}\right)^{\mathrm{T}} \\
\Xi C P^{\mathrm{T}} & -\widetilde{\mathrm{Q}}
\end{array}\right]<0 \quad i<j, i, j=1,2,}
\end{gathered}
$$

where $\Psi_{i j}=P A_{i}^{\mathrm{T}}+Y_{j}^{\mathrm{T}} B^{\mathrm{T}}+A_{i} P^{\mathrm{T}}+B Y_{j}-\alpha \Xi P^{\mathrm{T}}$ and $\widetilde{\mathrm{Q}}=$ $R^{-1 / 2} Q^{-1} R^{-1 / 2}$. Then, the desired state feedback gain can be chosen as $L_{j}=Y_{j} P^{-\mathrm{T}}$.

Proof. Considering Theorem 11 and the closed-loop system (49), this proof follows a similar way with the proof in Theorem 12, and it is easy to obtain the previous results. This completes the proof of the theorem.

Remark 14. The state feedback control method can be implemented by the management of the open resource so that the biological population can be controlled at the bounded range, and those unfavorable phenomena will be eliminated. In our daily life, managers can take action, such as adjusting revenue, drawing out favorable policy to encourage fishery, and abating pollution, in order to control the population density and economic profit.

\section{Numerical Examples}

To illustrate the results obtained, let us consider the following particular cases. We choose the ecological parameters according to biological sardine data of the Moroccan western coastline area, and these parameters are given in [6].

Example 1. Consider finite-time stabilization problem of the biological economic model, and select the ecological parameters as $r=0.015, k=1702.8, E=0.008, s=0.005$, and $c=200, a=800$ in appropriate units. Then, the closedloop system (48) can be described with the following data:

$$
\begin{gathered}
\Xi=\left[\begin{array}{lll}
1 & 0 & 0 \\
0 & 1 & 0 \\
0 & 0 & 0
\end{array}\right], \quad A_{1}=\left[\begin{array}{ccc}
0.0220 & 0.0000 & 0.0000 \\
-0.0000 & -0.0050 & 0.0000 \\
4.8000 & -13.6224 & -1.0000
\end{array}\right], \\
A_{2}=\left[\begin{array}{ccc}
-0.0080 & 0.0000 & 0.0000 \\
-0.0000 & -0.0050 & 0.0000 \\
4.8000 & 13.6224 & -1.0000
\end{array}\right], \quad B=\left[\begin{array}{lll}
1 & 0 & 0
\end{array}\right]^{\mathrm{T}} .
\end{gathered}
$$

Let $\alpha=0.001, c_{1}=10000, c_{2}=10000000, T=1000, R=I$. By Theorem 12, we can calculate that

$$
\begin{gathered}
P=10^{5} \times\left[\begin{array}{ccc}
0.0145 & 0.0003 & 0.0463 \\
0.0003 & 0.0002 & 0.0016 \\
0 & 0 & 1.8983
\end{array}\right], \\
Q=\left[\begin{array}{ccc}
0.0007 & -0.0014 & 0 \\
-0.0014 & 0.0580 & 0 \\
0 & 0 & 0.0294
\end{array}\right],
\end{gathered}
$$

$$
\begin{aligned}
& L_{1}=\left[\begin{array}{lll}
-36.6991 & 69.2352 & -0.0098
\end{array}\right], \\
& L_{2}=\left[\begin{array}{lll}
-36.6538 & 69.2506 & -0.0147
\end{array}\right] .
\end{aligned}
$$

So, we claim that $X^{\mathrm{T}}(t) \Xi^{\mathrm{T}} R \Xi X(t)<10000000, \forall t \quad \epsilon$ $[0,1000]$. The trajectory is shown in Figure 2.

Figure 1 shows the state trajectory of the open-loop biological economic system when $t \in[0,1000]$. Figure 2 shows the state trajectory of the closed-loop biological economic system with state feedback controller (47) when $t \in$ $[0,1000]$.

Example 2. Consider finite-time stabilization problem of the biological economic model with the white noise in Example 1. $\sigma^{2}$ is intensities of the white noise, and let $\sigma^{2}=0.01$. Select the ecological parameters as $r=0.015, k=1702.8, E=0.008$, $s=0.005, c=200$, and $a=800$ in appropriate units. The closed-loop system (49) can be formulated with the following parameters:

$$
\begin{aligned}
\Xi=\left[\begin{array}{lll}
1 & 0 & 0 \\
0 & 1 & 0 \\
0 & 0 & 0
\end{array}\right], & A_{1}=\left[\begin{array}{ccc}
0.0220 & 0.0000 & 0.0000 \\
-0.0000 & -0.0050 & 0.0000 \\
4.8000 & -13.6224 & -1.0000
\end{array}\right], \\
B=\left[\begin{array}{lll}
1 & 0 & 0
\end{array}\right]^{\mathrm{T}}, & A_{2}=\left[\begin{array}{ccc}
-0.0080 & 0.0000 & 0.0000 \\
-0.0000 & -0.0050 & 0.0000 \\
4.8000 & 13.6224 & -1.0000
\end{array}\right], \\
C & =\left[\begin{array}{ccc}
0.1 & 0 & 0 \\
0 & 0 & 0 \\
0 & 0 & 0
\end{array}\right] .
\end{aligned}
$$

Let $\alpha=0.001, c_{1}=10000, c_{2}=10000000, T=1000$, and $R=I$. By Theorem 13, we can calculate that

$$
\begin{gathered}
P=10^{5} \times\left[\begin{array}{ccc}
0.0837 & 0.0020 & 0.2679 \\
0.0020 & 0.0004 & 0.0098 \\
0 & 0 & 3.7772
\end{array}\right], \\
Q=\left[\begin{array}{ccc}
0.0001 & -0.0007 & 0 \\
-0.0007 & 0.0302 & 0 \\
0 & 0 & 0.0049
\end{array}\right], \\
L_{1}=\left[\begin{array}{lll}
-8.6579 & 47.2591 & -0.0345
\end{array}\right], \\
L_{2}=\left[\begin{array}{lll}
-8.6221 & 47.2785 & -0.0364
\end{array}\right]
\end{gathered}
$$

So, we claim that $\varepsilon\left\{X^{\mathrm{T}}(t) \Xi^{\mathrm{T}} R \Xi X(t)\right\}<10000000$, for all $t \epsilon$ $[0,1000]$. The trajectory is shown in Figure 4.

Figure 3 shows the state trajectory of the open-loop stochastic singular biological economic system with the white noise $\sigma^{2}$. Taking $\sigma^{2}=0.01$, we know that the population density and the unit price are not stable over the finite-time interval $[0,1000]$. The economic profit is random fluctuation in Figure 3, because the economic profit is affected by the unit price of the population and the population density of our proposed biological economic model in the random disturbance environment. 


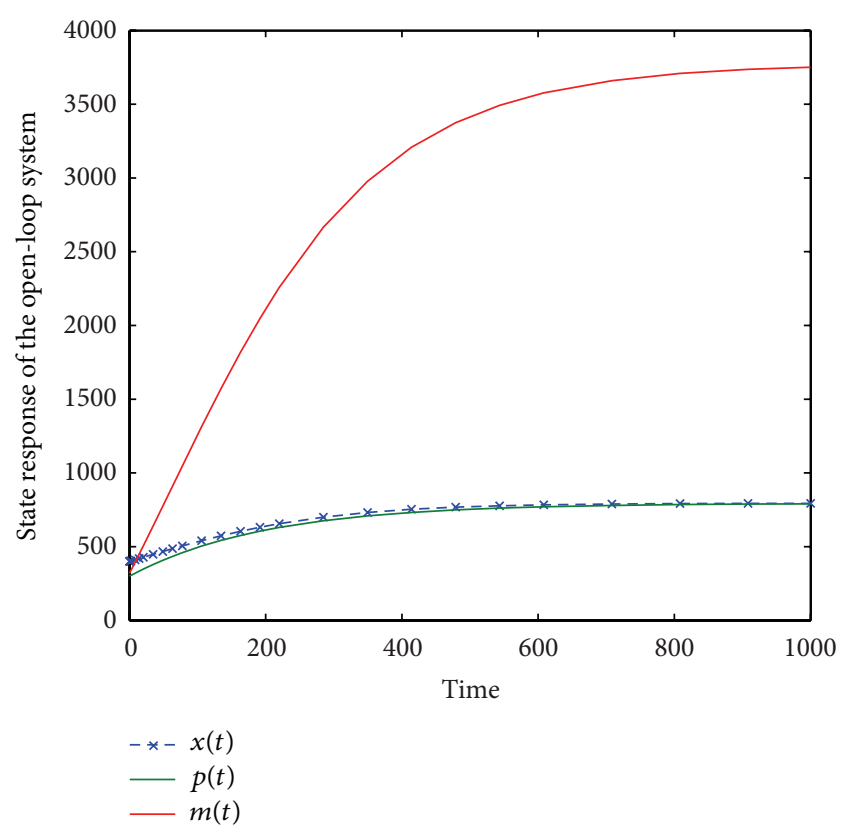

FIGURE 1: Trajectory of the open-loop biological economic system.

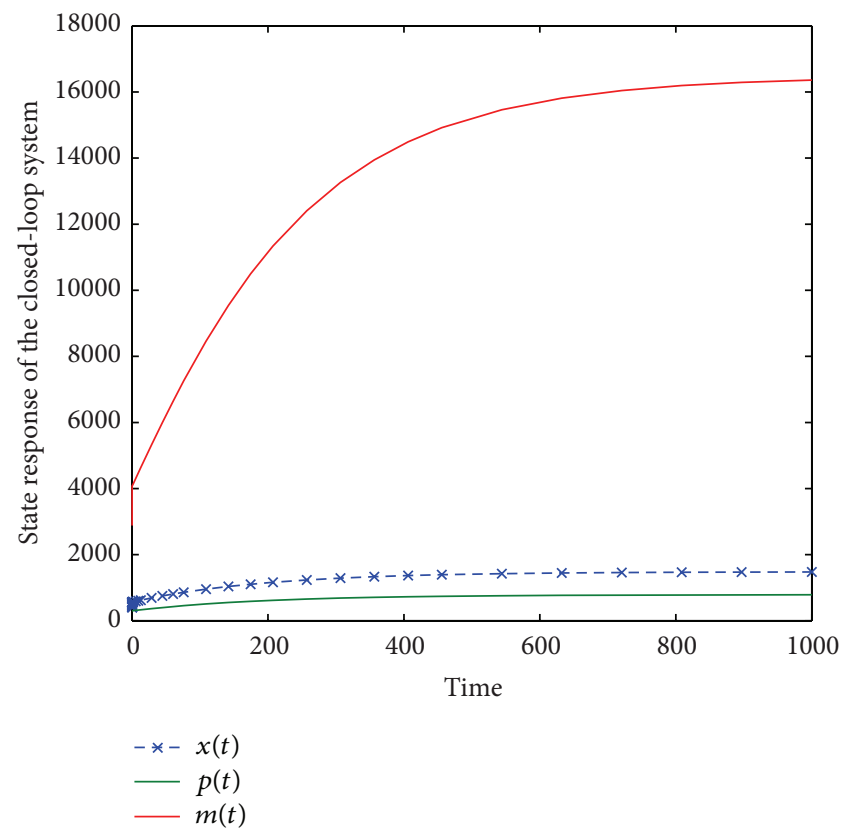

FIGURE 2: Trajectory of the closed-loop biological economic system.

Figure 4 shows the state trajectory of the closed-loop stochastic singular biological economic system with the white noise $\sigma^{2}=0.01$ through the state feedback controller (47). From Figure 4, it can be seen that the economic profit tends to be stable over the finite-time interval $[0,1000]$.

\section{Conclusions}

In this paper, we study the problems of finite-time stability and control for a class of stochastic singular biological economic systems based on T-S fuzzy model. By the theorems

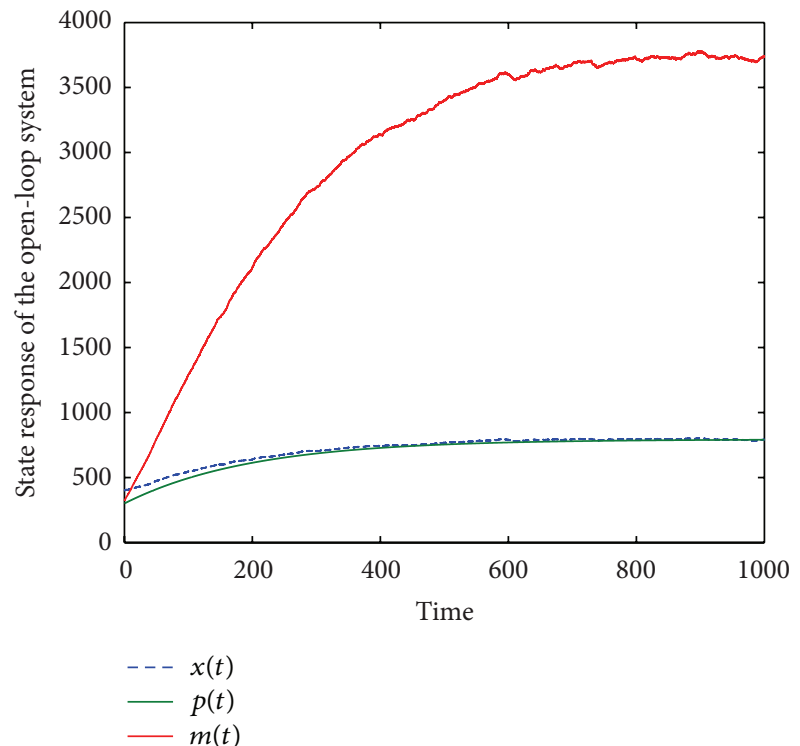

FIGURE 3: Trajectory of the open-loop stochastic singular system.

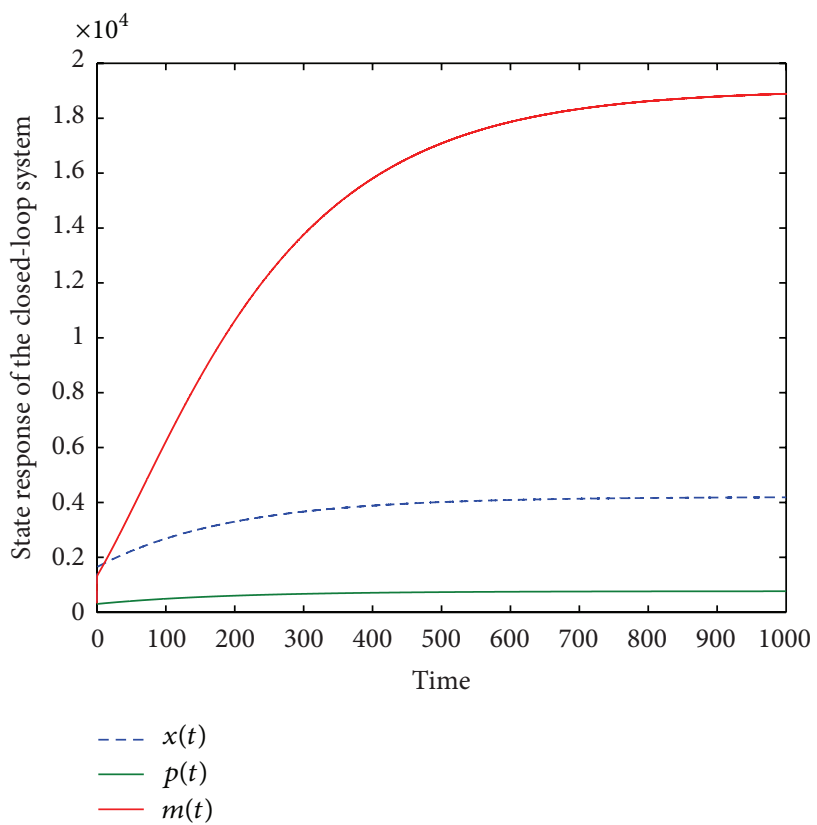

FIgURE 4: Trajectory of the closed-loop stochastic singular system.

we presented, some novel sufficient conditions are derived which ensure that the systems are stable in finite time. And then, the corresponding controller design methods are obtained. Finally, numerical examples are presented to illustrate the effectiveness of the proposed results. Our proposed results in this paper can also be applied to the study of other classes of systems.

\section{Acknowledgment}

This work was supported by National Natural Science Foundation of China under Grant no. 60974004 and no. 61273008. 


\section{References}

[1] C. W. Clark, Mathematical Bioeconomics: The Optimal Management of Renewable Resources, Pure and Applied Mathematics, John Wiley \& Sons, New York, NY, USA, 2nd edition, 1990.

[2] M. Jerry and N. Raïssi, "A policy of fisheries management based on continuous fishing effort," Journal of Biological Systems, vol. 9, no. 4, pp. 247-254, 2001.

[3] M. Jerry and N. Raïssi, "The optimal strategy for a bioeconomical model of a harvesting renewable resource problem," Mathematical and Computer Modelling, vol. 36, no. 11-13, pp. 1293-1306, 2002.

[4] R. Q. Grafton, L. K. Sandal, and S. I. Steinshamn, "How to improve the management of renewable resources: the case of Canada's northern cod fishery," American Journal of Agricultural Economics, vol. 82, no. 3, pp. 570-580, 2000.

[5] M. B. Schäefer, "Some aspects of the dynamics of populations important to the management of the commercial marine fisheries," Bulletin of Mathematical Biology, vol. 53, no. 1-2, pp. 253-279, 1991.

[6] C. Jerry and N. Raïssi, "Can management measures ensure the biological and economical stabilizability of a fishing model?" Mathematical and Computer Modelling, vol. 51, no. 5-6, pp. 516526, 2010.

[7] C. Bernstein, P. Auger, and J. C. Poggiale, "Predator migration decisions, the ideal free distribution, and predator-prey dynamics," American Naturalist, vol. 153, no. 3, pp. 267-281, 1999.

[8] J. T. Lafrance, "Linear demand functions in theory and practice," Journal of Economic Theory, vol. 37, no. 1, pp. 147-166, 1985.

[9] C. Mullon and P. Fréon, "Prototype of an integrated model of the worldwide system of small pelagic fisheries," in Climate Change and the Economics of the World's Fisheries: Examples of Small Pelagic Stocks, New Horizons in Environmental Economics, pp. 262-295, Edward Elgar, Cheltenham, UK, 2005.

[10] L. Lasselle, S. Svizzero, and C. Tisdell, "Stability and cycles in a cobweb model with heterogeneous expectations," Macroeconomic Dynamics, vol. 9, no. 5, pp. 630-650, 2005.

[11] S. Ayasun, C. O. Nwankpa, and H. G. Kwatny, "Computation of singular and singularity induced bifurcation points of differential-algebraic power system model," IEEE Transactions on Circuits and Systems. I. Regular Papers, vol. 51, no. 8, pp. 15251538, 2004.

[12] R. Riaza, "Singularity-induced bifurcations in lumped circuits," IEEE Transactions on Circuits and Systems. I, vol. 52, no. 7, pp. 1442-1450, 2005.

[13] D. G. Luenberger, "Nonlinear descriptor systems," Journal of Economic Dynamics \& Control, vol. 1, no. 3, pp. 219-242, 1979.

[14] H. Krishnan and N. H. McClamroch, "Tracking in nonlinear differential-algebraic control systems with applications to constrained robot systems," Automatica, vol. 30, no. 12, pp. 18851897, 1994.

[15] A. M. Bloch, M. Reyhanoglu, and N. H. McClamroch, "Control and stabilization of nonholonomic dynamic systems," IEEE Transactions on Automatic Control, vol. 37, no. 11, pp. 1746-1757, 1992.

[16] H. S. Gordon, "Economic theory of a common property resource: the fishery," The Journal of Political Economy, vol. 62, no. 2, pp. 124-142, 1954.

[17] X. Zhang, Q.-1. Zhang, and Y. Zhang, "Bifurcations of a class of singular biological economic models," Chaos, Solitons and Fractals, vol. 40, no. 3, pp. 1309-1318, 2009.
[18] C. Liu, Q. Zhang, Y. Zhang, and X. Duan, "Bifurcation and control in a differential-algebraic harvested prey-predator model with stage structure for predator," International Journal of Bifurcation and Chaos, vol. 18, no. 10, pp. 3159-3168, 2008.

[19] Y. Zhang and Q. L. Zhang, "Bifurcations and control in singular biological economic model with stage structure," Journal of Systems Engineering, vol. 22, no. 3, pp. 233-238, 2007.

[20] Q. Zhang, C. Liu, and X. Zhang, Complexity, Analysis and Control of Singular Biological Systems, vol. 421 of Lecture Notes in Control and Information Sciences, Springer, London, UK, 2012.

[21] R. M. May, Stability and Complexity in Model Ecosystems, Princeton University Press, NJ, USA, 1973.

[22] M. Carletti, "Numerical simulation of a Campbell-like stochastic delay model for bacteriophage infection," Mathematical Medicine and Biology, vol. 23, no. 4, pp. 297-310, 2006.

[23] X. Mao, G. Marion, and E. Renshaw, "Environmental Brownian noise suppresses explosions in population dynamics," Stochastic Processes and their Applications, vol. 97, no. 1, pp. 95-110, 2002.

[24] C. Ji, D. Jiang, and N. Shi, "Analysis of a predator-prey model with modified Leslie-Gower and Holling-type II schemes with stochastic perturbation," Journal of Mathematical Analysis and Applications, vol. 359, no. 2, pp. 482-498, 2009.

[25] C. Y. Ji, D. Jiang, and X. Li, "Qualitative analysis of a stochastic ratio-dependent predator-prey system," Journal of Computational and Applied Mathematics, vol. 235, no. 5, pp. 1326-1341, 2011.

[26] C. Ji and D. Jiang, "Dynamics of a stochastic density dependent predator-prey system with Beddington-DeAngelis functional response," Journal of Mathematical Analysis and Applications, vol. 381, no. 1, pp. 441-453, 2011.

[27] J. Lv and K. Wang, "Asymptotic properties of a stochastic predator-prey system with Holling II functional response," Communications in Nonlinear Science and Numerical Simulation, vol. 16, no. 10, pp. 4037-4048, 2011.

[28] Z. W. Liu, N. Z. Shi, D. Q. Jiang, and C. Y. Ji, “The asymptotic behavior of a stochastic predator-prey system with holling II functional response," Abstract and Applied Analysis, vol. 2012, Article ID 801812, 14 pages, 2012.

[29] X. Song, H. Zhang, and L. Xie, "Stochastic linear quadratic regulation for discrete-time linear systems with input delay," Automatica, vol. 45, no. 9, pp. 2067-2073, 2009.

[30] B.-S. Chen and W. Zhang, "Stochastic $H_{2} / H_{\infty}$ control with state-dependent noise," IEEE Transactions on Automatic Control, vol. 49, no. 1, pp. 45-57, 2004.

[31] P. Doroto, "Short time stability in linear time-varying systems," Proceeding of IRE International Convention Record, vol. 4, pp. 83-87, 1961.

[32] F. Amato, M. Ariola, and P. Dorato, "Finite-time control of linear systems subject to parametric uncertainties and disturbances," Automatica, vol. 37, no. 9, pp. 1459-1463, 2001.

[33] F. Amato and M. Ariola, "Finite-time control of discrete-time linear systems," IEEE Transactions on Automatic Control, vol. 50, no. 5, pp. 724-729, 2005.

[34] L. Liu and J. Sun, "Finite-time stabilization of linear systems via impulsive control," International Journal of Control, vol. 81, no. 6, pp. 905-909, 2008.

[35] S. Zhao, J. Sun, and L. Liu, "Finite-time stability of linear timevarying singular systems with impulsive effects," International Journal of Control, vol. 81, no. 11, pp. 1824-1829, 2008.

[36] W. Zhang and X. An, "Finite-time control of linear stochastic systems," International Journal of Innovative Computing, Information and Control, vol. 4, no. 3, pp. 689-696, 2008. 
[37] T. Takagi and M. Sugeno, "Fuzzy identification of systems and its applications to modeling and control," IEEE Transactions on Systems, Man and Cybernetics, vol. 15, no. 1, pp. 116-132, 1985.

[38] S. G. Hu, B. M. Huang, and F. K. Wu, Stochastic Differential Equations, Science Press, Beijing, China, 2008.

[39] B. Oksendal, Stochastic Differential Equations: An Introduction with Applications, Springer, New York, NY, USA, 5th edition, 2000.

[40] Y. Zhang, C. Liu, and X. Mu, "Robust finite-time $H_{\infty}$ control of singular stochastic systems via static output feedback," Applied Mathematics and Computation, vol. 218, no. 9, pp. 5629-5640, 2012. 


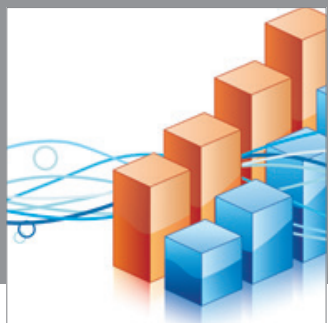

Advances in

Operations Research

mansans

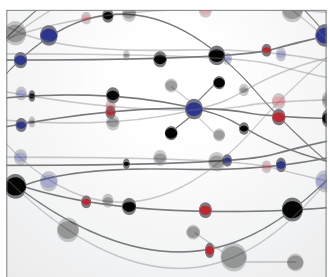

The Scientific World Journal
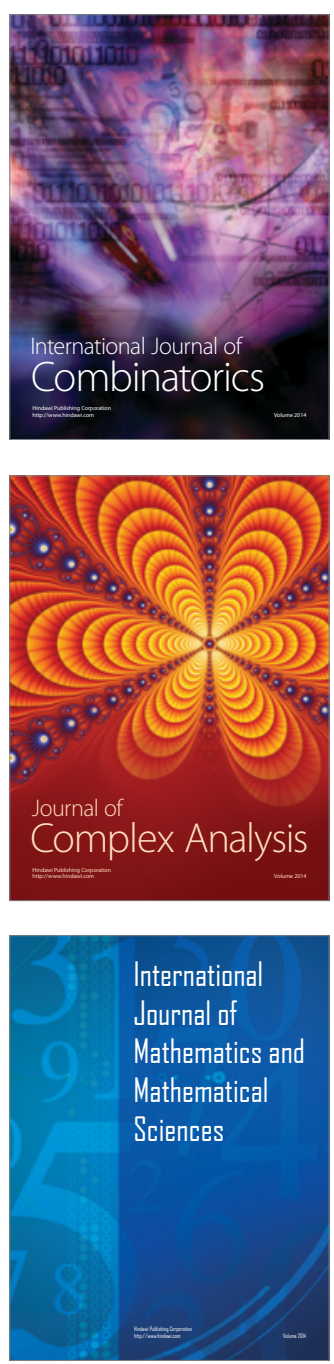
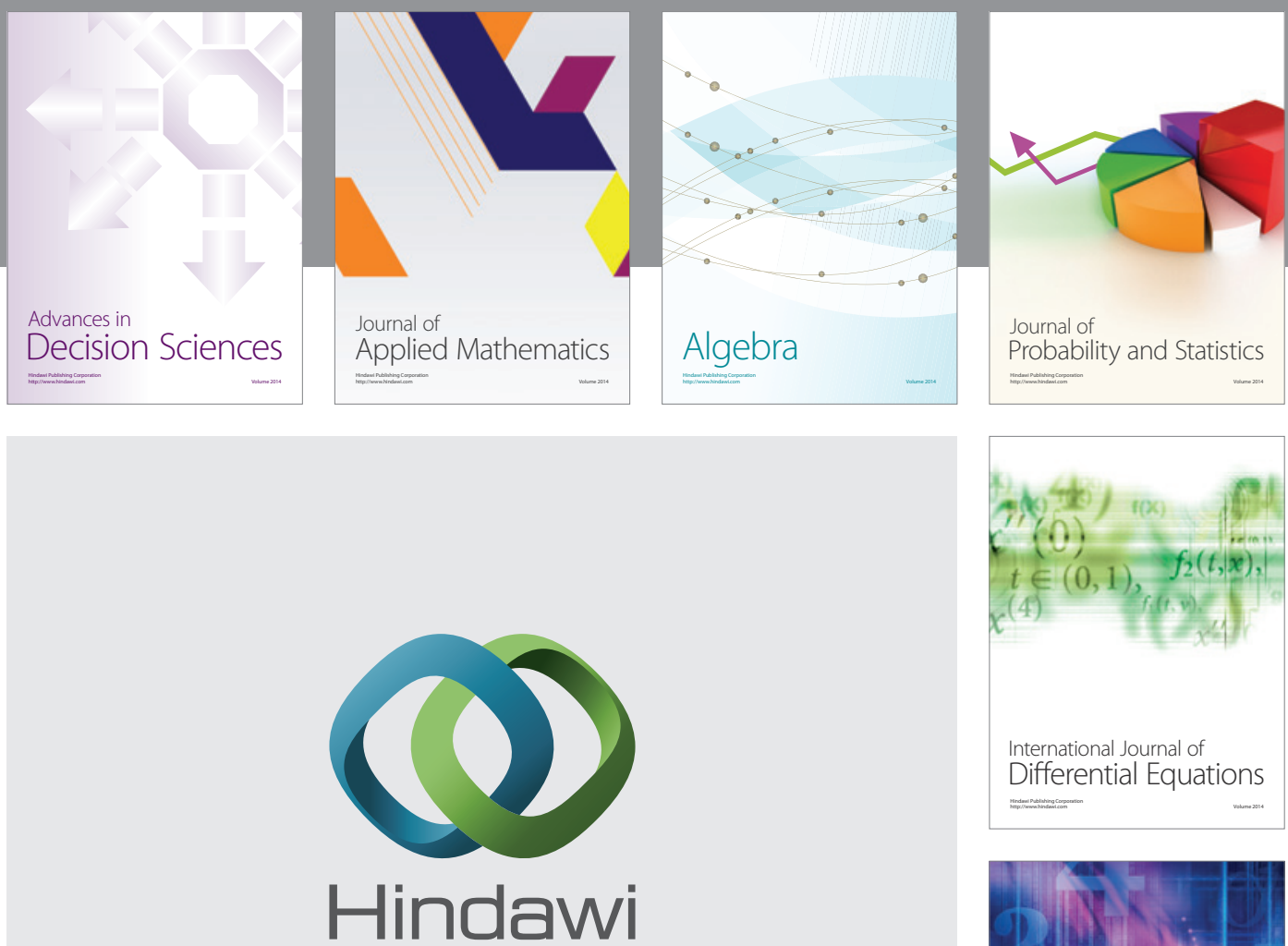

Submit your manuscripts at http://www.hindawi.com
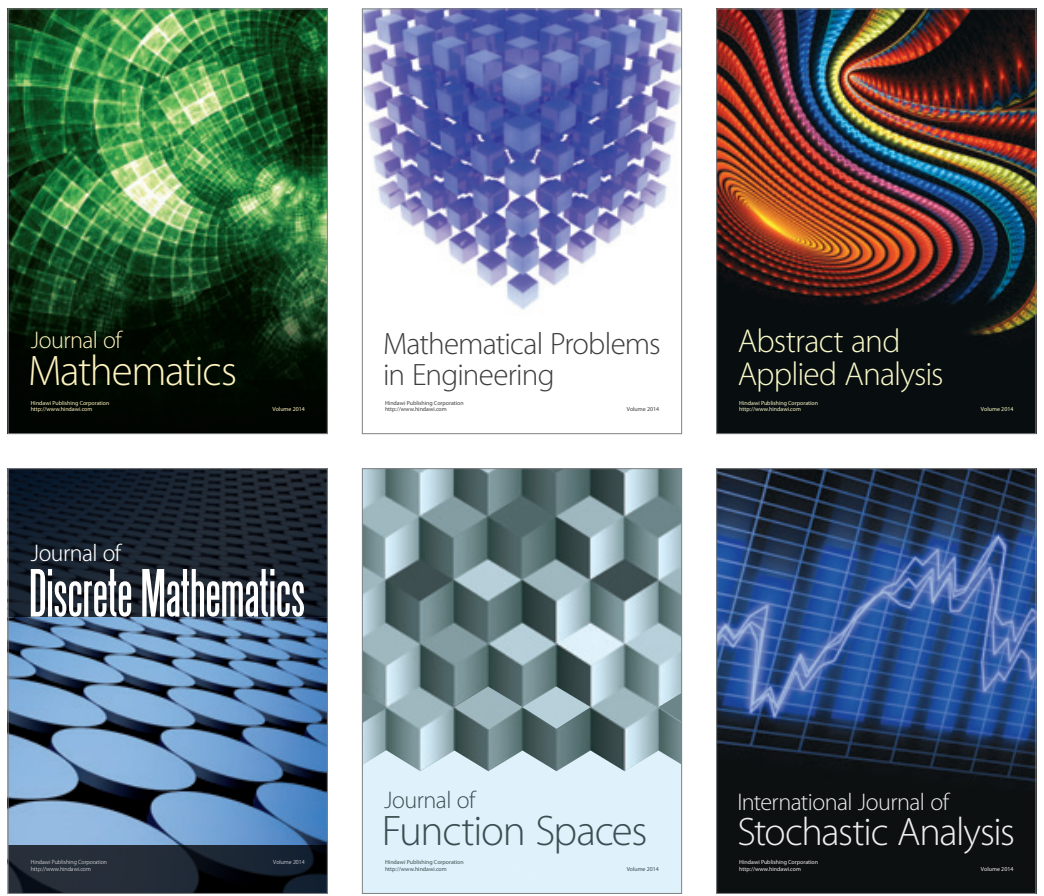

Journal of

Function Spaces

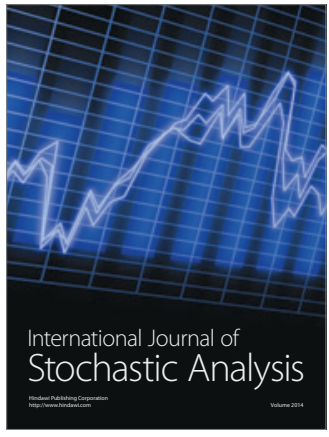

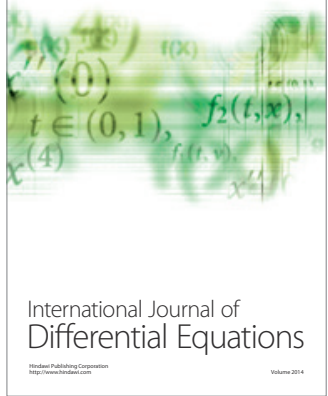
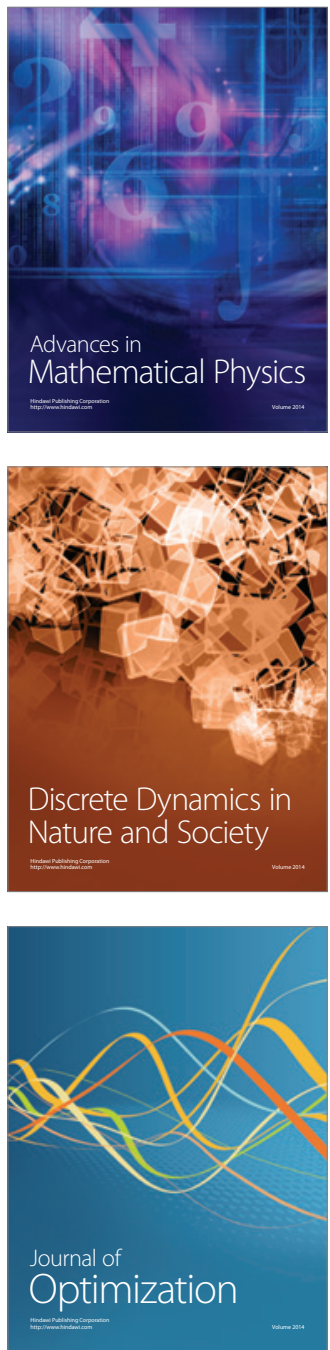